\title{
Electro-Absorption Modulator Integrated Lasers With Enhanced Signal Injection Efficiency
}

\author{
Cheng Guan Lim, Member, IEEE
}

\begin{abstract}
By incorporating a lumped-element impedancematching network on the laser diode carrier/submount, the signal injection efficiency of electro-absorption modulator integrated lasers is significantly improved through the reduction of input return loss. Over a frequency range spanning from 5.8 to $10 \mathrm{GHz}$, an improvement of approximately $12 \%$ to $37 \%$ in signal injection efficiency is observed. To avoid increasing the component-count, a unique implementation of the lumped element impedance matching network is proposed.
\end{abstract}

Index Terms-Electro-absorption modulator integrated lasers, impedance matching, input return loss.

\section{INTRODUCTION}

$\mathbf{T}$ HE ADVENT of optical fiber communications to accommodate the ever-increasing demand on channel transfer-rate and transmission capacity has led to perpetual interest in dense wavelength division multiplexing (DWDM) [1]. 10-Gb/s optical fiber communication systems have been deployed in parts of the communication infrastructure to provide a higher channel transfer-rate and larger transmission capacity. In the past, Mach-Zehnder modulators (MZM) have been used in sectors where the required transmission rate and range are beyond the capability of directly modulated laser diodes [2], [3]. Since the introduction of the relatively cheaper electro-absorption modulator (EAM) [4] and electro-absorption modulator integrated lasers (EML) [5], these devices have replaced the MZM in some of the segments where EAMs and EMLs are applicable [6]-[8]. Besides costing less than the MZM and can be monolithically integrated with a distributed-feedback (DFB) laser diode (LD), the EAM has the advantage of operating at a significantly lower voltage [9]. Nevertheless, one tedious problem associated with EAMs and EMLs is the large impedance mismatch between these devices and the signal source. Typical 10-Gb/s EMLs exhibit an input return loss $\left(S_{11}\right)$ of more than $-8 \mathrm{~dB}$ and $-10 \mathrm{~dB}$ for frequencies above approximately 5 and $8 \mathrm{GHz}$, and a $-3 \mathrm{~dB}$ modulation bandwidth $\left(S_{21}\right)$ ranging from 8 to $11 \mathrm{GHz}$. Although some of these EMLs have sufficient bandwidth to meet the specified bandwidth (i.e., more than $10 \mathrm{GHz}$ ), their input return loss falls short of the specified value (i.e., less than $-10 \mathrm{~dB}$ for frequency up to more than $10 \mathrm{GHz}$ ). To-date,

Manuscript received July 30, 2007; revised November 17, 2007.

The author was with the Department of Photonics/Institute of Electro-Optical Engineering, National Chiao Tung University, Hsinchu 30010, Taiwan, R.O.C. $\mathrm{He}$ is now with the Ultrafast Photonic Devices Laboratory, National Institute of Advanced Industrial Science and Technology (AIST), Tsukuba, Ibaraki 305568, Japan (e-mail: c.g.lim@ieee.org).

Digital Object Identifier 10.1109/JLT.2007.915275 two methods of designing EML modules to meet the required specifications have been proposed [10], [11]. To reduce the input return loss, Ishimura et al. [10] have proposed to use a current-compensation technique to control the impedance seen by the signal source, whereas Lim et al. [11] have proposed a reactive impedance matching technique to trim the reactive part of the impedance seen by the signal source. The unfavorable aspect of these two methods is that the device capacitance of the EAM section of the EML must be reduced to not more than approximately $0.4 \mathrm{pF}$; causing optical performance to be compromised. The tradeoff between a low device capacitance and a good optical performance (e.g., extinction ratio, chirp, optical output power) has made it difficult to achieve the radio-frequency $(\mathrm{RF})$ and optical performance specifications simultaneously. A typical 10-Gb/s EML design with an optimum optical performance has a device capacitance of approximately $0.6 \mathrm{pF}$ for the EAM section. Although a difference of $0.2 \mathrm{pF}$ is small in the general domain, it is very significant in the high-frequency (HF) domain. In fact, this is a large value (a difference of approximately $33 \%$ ) considering the physical size of the EAM, and its impact on the performance of the EAM is significant. To relief the above problem, a method (similar to that proposed in [12]) to improve the signal injection efficiency and optical performance of $10-\mathrm{Gb} / \mathrm{s}$ EMLs will be proposed in this paper. In contrast to [12], a systematic lumped-element impedance-matching network (L-network) design procedure is followed and implementation method and design equations will be presented in this paper. Moreover, the "+" design proposed in [12] functions more like staggered-step impedance transformer or open-circuit stubs which consist of two impedance discontinuities that cause signal reflections and result in signal distortion and degraded jitter. Furthermore, using two discrete sections to introduce the required capacitance and inductance restrict the implementation to bit-rates lower than $10 \mathrm{~Gb} / \mathrm{s}$ because parasitic inductance and capacitance are introduced by these sections. In this paper, a unique implementation to show how this impedance-matching technique that originally used lumped-elements can be applied to higher frequencies in excess of that demonstrated in [12]. This unique implementation that uses only one microstrip transmission-line section instead of two (as in [12]) to implement the L-network is expected to have better signal quality and jitter.

\section{DESIGN OF THE IMPEDANCE-MATCHING ELEMENT}

Fig. 1(a) shows the schematic drawing of a typical design of an EML, and Fig. 1(b) shows the structure details of the EAM section. Based on this device structure of the EML, an equivalent electrical circuit model of the EML was derived [Fig. 1(c)] 


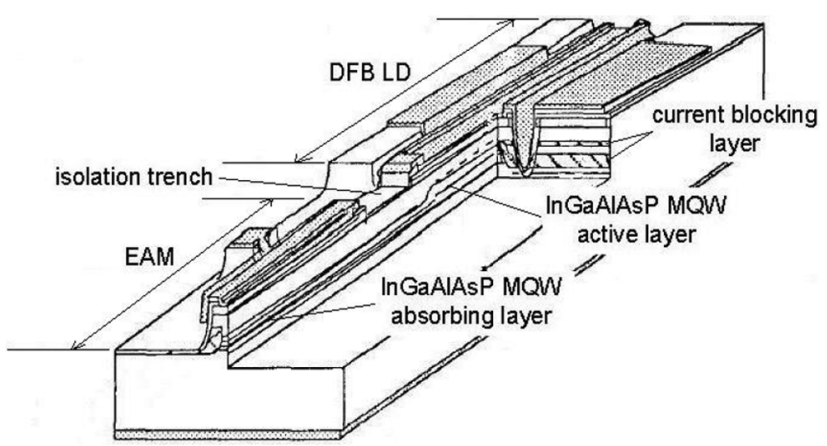

(a)

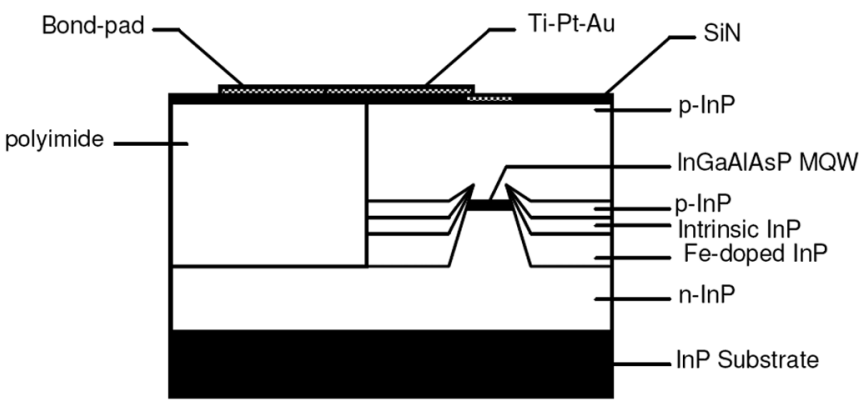

(b)

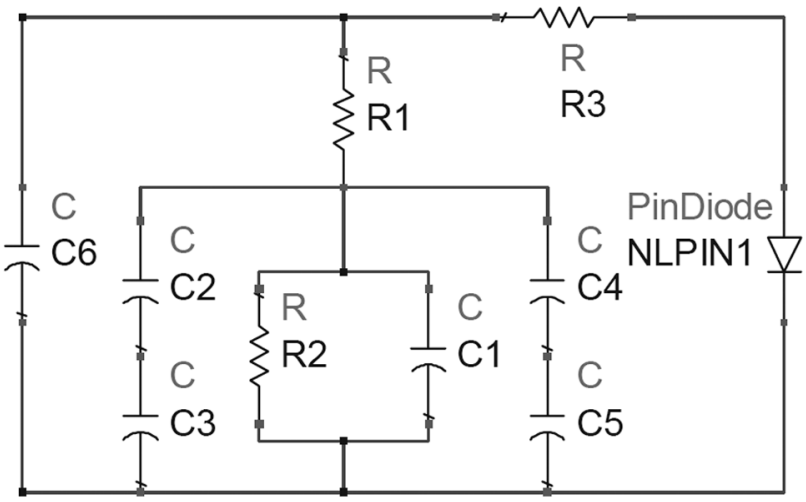

(c)

Fig. 1. Schematic diagram of the (a) electro-absorption modulator integrated laser, (b) device structure of the electro-absorption modulator section, and (c) equivalent electrical circuit model of the EML.

where the laser diode is represented by a $P-I-N$ diode. Using the dimensions of a typical EAM design, each of the model parameters was calculated and extracted from $S_{11}$ and $S_{21}$ responses. The extracted values for the device resistance (R1), differential resistance (R2), isolation resistance (R3), and capacitance of the absorption layer (C1), intrinsic layers (C2 and $\mathrm{C} 4)$, iron-doped layers (C3 and $\mathrm{C} 5$ ), and polyimide layer (C6) are $5 \Omega, 300 \Omega, 20 \mathrm{k} \Omega, 0.42 \mathrm{pF}, 0.732 \mathrm{pF}, 0.082 \mathrm{pF}$, and $0.085 \mathrm{pF}$, respectively.

On a module level, the equivalent electrical circuit model of an EML module is shown in Fig. 2(a) where the signal source impedance is represented by a $50-\Omega$ termination, the signal feeder on the seven-pin butterfly package for soldering the signal lead of the RF connector is represented by a co-planar waveguide transmission-line CPW1, the signal feeder on the $\mathrm{LD}$ carrier/submount where the EML is bonded is represented by a microstrip transmission-line TL1, R4 acts as a termination resistor to pull the impedance of the EAM close to the

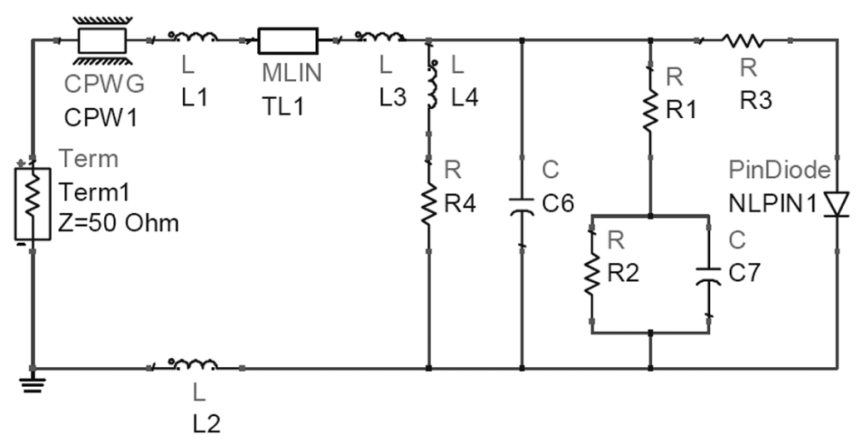

(a)

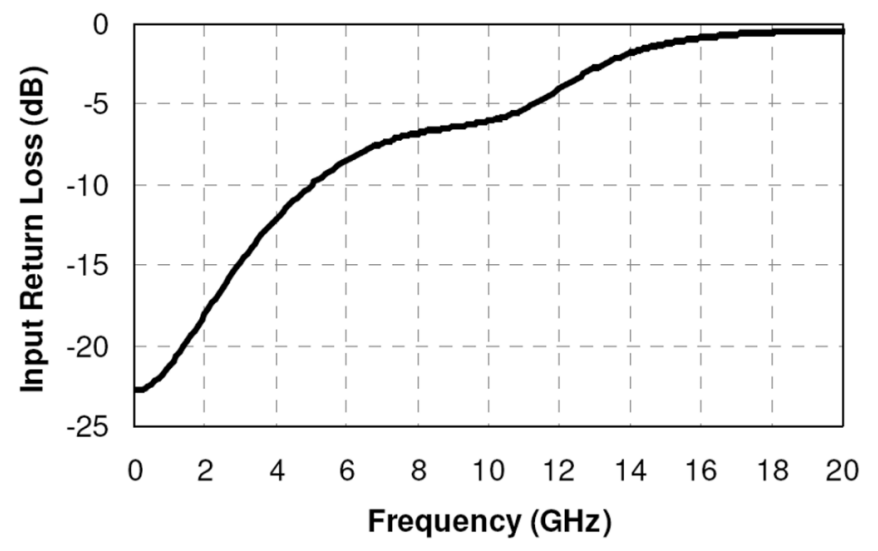

(b)

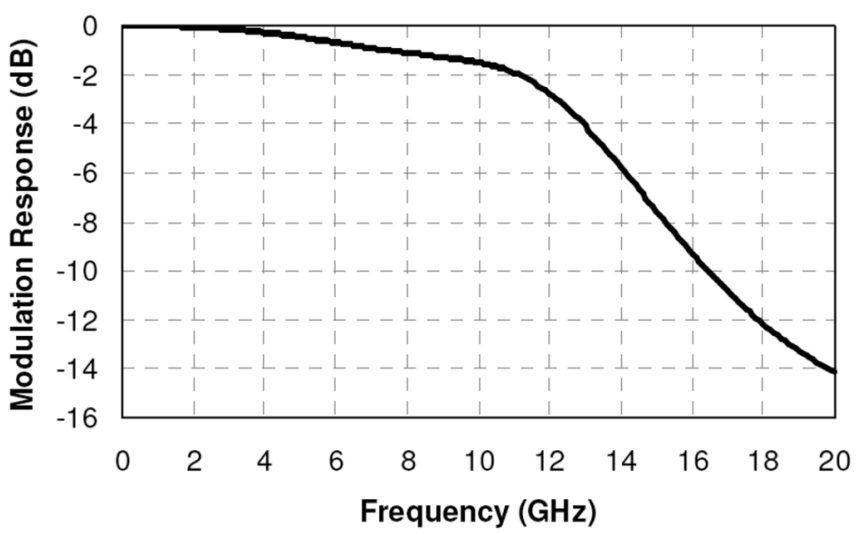

(c)

Fig. 2. (a) Equivalent electrical circuit model of the EML module. (b) Input return loss response. (c) Modulation response of the EML module.

characteristic impedance of the signal source, the equivalent capacitance of $\mathrm{C} 1, \mathrm{C} 2, \mathrm{C} 3, \mathrm{C} 4$, and $\mathrm{C} 5$ as in Fig. 1(c) is represented by $\mathrm{C} 7$, the bonding-wire that connects the signal line of CPW1 to TL1, the ground plane of CPW1 to the ground plane of TL1, the signal line of TL1 to the bond-pad of the EAM, the bond-pad of the EAM to the termination resistor are represented by $\mathrm{L} 1, \mathrm{~L} 2, \mathrm{~L} 3$, and $\mathrm{L} 4$, respectively. The value of L1, L2, L3, and L4 used in this work are 0.3, 0.3, 0.5, and $0.5 \mathrm{nH}$, respectively. These are very reasonable and practical values to consider. Typically, CPW1 and TL1 are designed to have a characteristic impedance of $50 \Omega$. Taking all the above into consideration, the input return loss response and modulation response obtained from the well-established RF simulator (Advanced Design System) developed by Agilent Technologies are shown in Fig. 2(b) and (c), respectively. It 


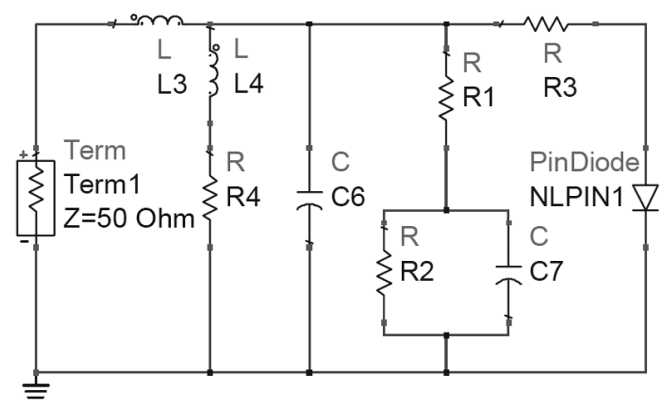

(a)

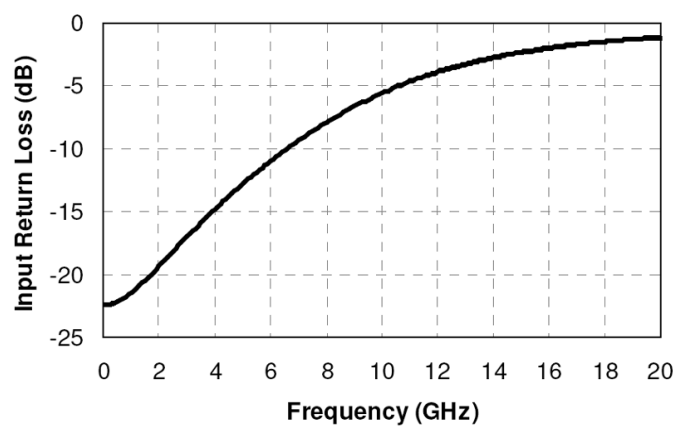

(b)

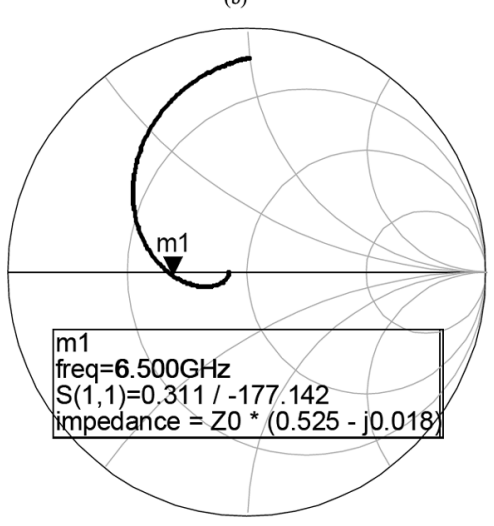

(c)

Fig. 3. (a) Portion of the equivalent electrical circuit of the EML module that has been treated as the load. (b) Input return loss response. (c) Smith chart of the load.

was observed that the input return loss is greater than $-10 \mathrm{~dB}$ for frequencies above approximately $5 \mathrm{GHz}$. Although this design has a modulation bandwidth of approximately $12 \mathrm{GHz}$, its signal injection efficiency and signal quality are not good due to substantially high input reflection.

To improve the input return loss response of the EMLs, the L-network appears to be a very suitable candidate among the various impedance-matching techniques as it can achieve a good impedance-matching between a complex load and a microwave system [12]. To design an appropriate L-network, the load [Fig. 3(a)] impedance was noted (i.e., $26.25-j 0.9 \Omega$ ) at the frequency which the input return loss response crossed the $-10 \mathrm{~dB}$ mark (i.e., $6.5 \mathrm{GHz}$ ) and inserted into the following equations:

$$
\begin{aligned}
& X=\sqrt{R_{L}\left(Z_{0}-R_{L}\right)}-X_{L} \\
& B= \pm \frac{\sqrt{\frac{\left(Z_{0}-R_{L}\right)}{R_{L}}}}{Z_{0}}
\end{aligned}
$$

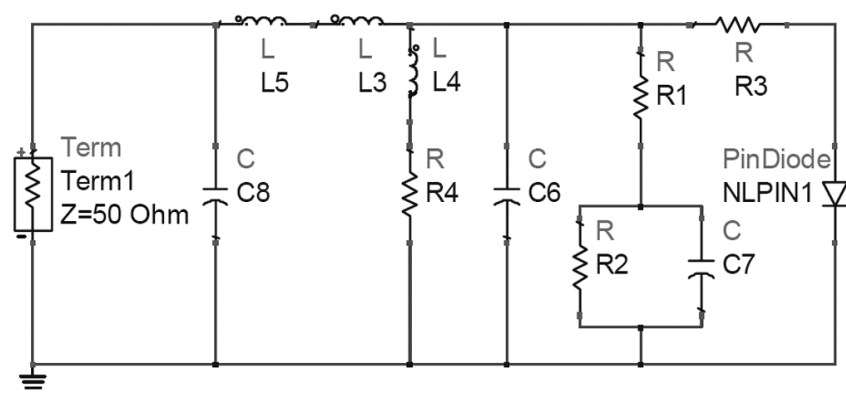

Fig. 4. Schematic diagram showing the incorporation of the L-network (L5 and C8) before the portion of the equivalent electrical circuit of the EML module that has been considered as the load.

where $X$ and $B$ are the reactance of the capacitor and inductor respectively, $R_{L}$ and $X_{L}$ are the real and imaginary parts of the load impedance, and $Z_{0}$ is the characteristic impedance of the transmission-line which the load is to be matched to. As a result, the value of L5 and C8 (Fig. 4) were found to be $0.633 \mathrm{nH}$ and $0.466 \mathrm{pF}$, respectively. As the value of the inductance and capacitance of the L-network are non-standard values, this adds on to the additional cost. To implement the L-network with no additional cost to the original cost, it is proposed here to implement the L-network using transmission-lines whereby no additional concern over reliability issue due to increased component-count is introduced. Here, the microstrip transmission-line has been used for illustration. The shunt capacitance $(C)$ and inductance $(L)$ of the microstrip transmission-line L-network are calculated as follows:

$$
\begin{aligned}
C & =\frac{\varepsilon_{0} \varepsilon_{r} A}{d} \\
L & =\frac{2 Z_{0} \tan \frac{\beta l}{2}}{2 \pi f}
\end{aligned}
$$

where $\varepsilon_{o}$ is the permittivity of free-space, $\varepsilon_{r}$ the relative permittivity of the substrate, $A$ the surface area of the transmission-line, $d$ the thickness of the substrate, $\beta$ the propagation constant, $l$ the length of the transmission-line, and $f$ the frequency of concern. The shunt capacitance and series inductance of various combinations of length and width of the microstrip transmission-line L-network were calculated and the one that gives the desired capacitance and inductance was used [Fig. 5(a)].

\section{Simulation Results AND EVAluation}

Consequently, results showed that an input return loss of below $-10 \mathrm{~dB}$ for frequencies below $9.5 \mathrm{GHz}$ and a modulation bandwidth of $11 \mathrm{GHz}$ have been achieved [Fig. 5(b) and (c)]. As the input return loss still falls short of the required value, the capacitance of the absorption layer was reduced, and it was found that the device capacitance of the EAM section must not be more than approximately $0.5 \mathrm{pF}$ in order for this approach to meet both specifications. With the device capacitance of the EAM section reduced, the input return loss of the load was noted to cross the $-10 \mathrm{~dB}$ mark at $7.7 \mathrm{GHz}$ [Fig. 6(a)], and the load impedance at this frequency was noted to be $26.25+j 1.85 \Omega$ [Fig. 6(b)]. With this impedance, the inductance and capacitance of the L-network were calculated 


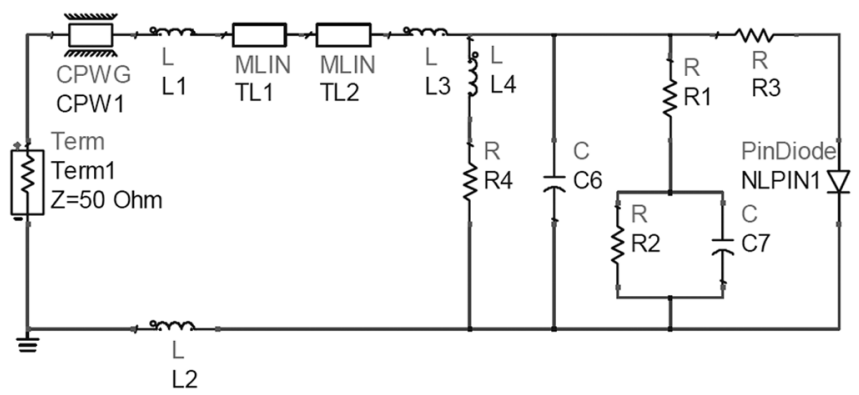

(a)

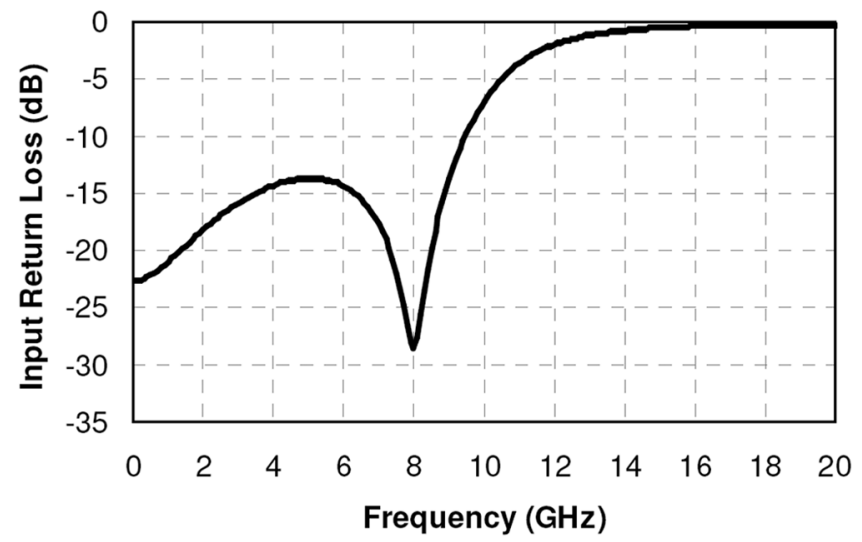

(b)

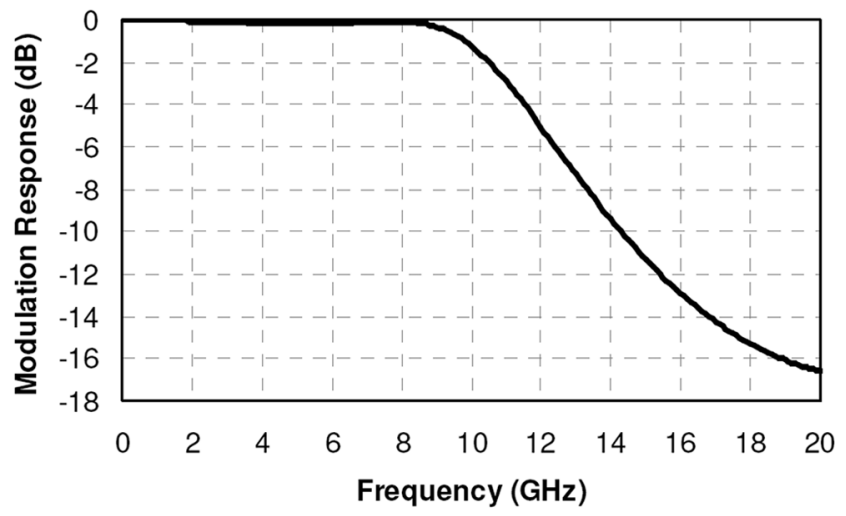

(c)

Fig. 5. (a) TL2 is the microstrip transmission-line implementation of the L-network shown in Fig. 4. The (b) input return loss response and (c) modulation responses of the $10 \mathrm{~Gb} / \mathrm{s}$ EML module incorporating the microstrip transmission-line implementation of the L-network.

to be $0.478 \mathrm{nH}$ and $0.393 \mathrm{pF}$, respectively. The shunt capacitance and series inductance of various combinations of length and width of the microstrip transmission-line L-network were calculated and the one that gives the desired capacitance and inductance was used. Consequently, results showed that the input return loss stays below $-10 \mathrm{~dB}$ up to a frequency of more than $10 \mathrm{GHz}$ and the modulation bandwidth is nearly $12 \mathrm{GHz}$ [Fig. 7(a) and (b)]. Compared to the typical design shown in Fig. 2(a), this design has significantly improved the signal injection efficiency of the EML from $60 \%-68 \%$ to $80 \%-97 \%$ over a frequency range spanning from 5.8 to $10 \mathrm{GHz}$. In comparison to the techniques proposed by Ishimura et al. [10] and Lim et al. [11], the method proposed in this work has the advantage of not having to reduce the device capacitance of the EAM drastically by approximately $33 \%$ which greatly

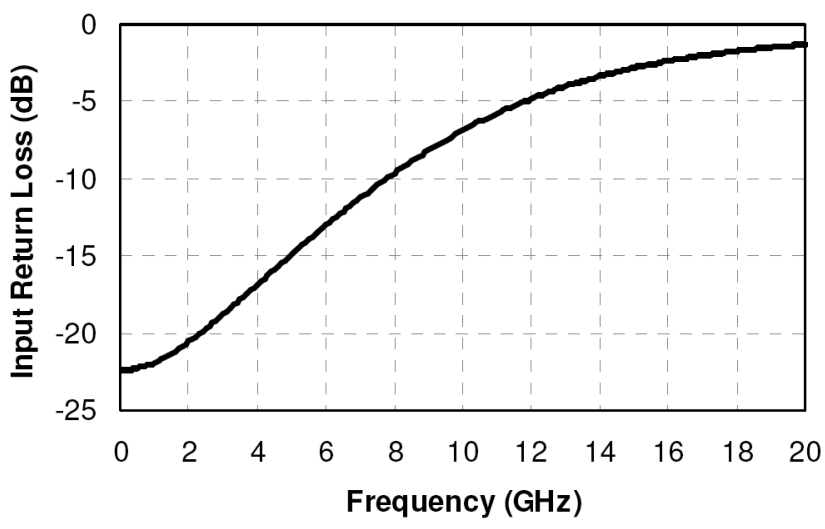

(a)

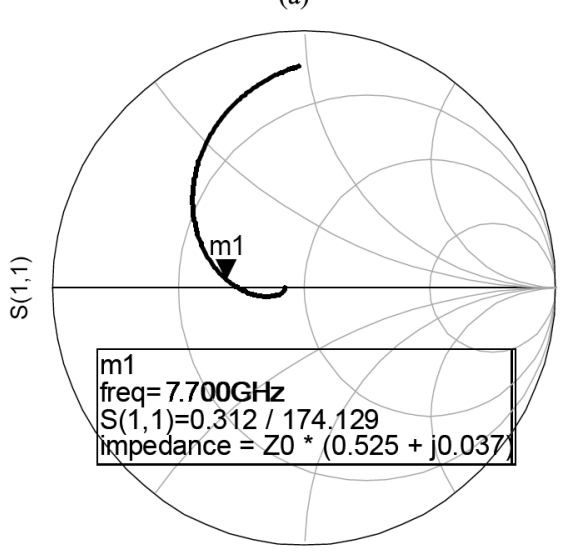

(b)

Fig. 6. (a) Input return loss response and (b) Smith chart of the load for the case whereby the device capacitance of the EAM section of the EML is reduced to approximately $0.5 \mathrm{pF}$.

affects the optical performance of the EAM. Although the design proposed in this work also resorts to reducing the device capacitance of the EAM (from approximately 0.6 to $0.5 \mathrm{pF}$ ), the amount (i.e., 14\%) is relatively small compared to $33 \%$. The improvement of the proposed design is more than twofold, hence relieving the compromise made on the optical performance by a factor of more than two. Considering the difficulty in achieving good signal injection efficiency and good optical performance simultaneously, this is indeed a significant and additional improvement.

To understand the mechanism responsible for the above improvement, the input impedance response of the proposed design [Fig. 5(a)] as well as that of the original design [Fig. 2(a) with device capacitance reduced to approximately $0.5 \mathrm{pF}]$ are plotted as shown in Fig. 8. With the incorporation of the L-network, it can be observed from Fig. 8(a) that the real part of the input impedance has been controlled to within $39 \Omega$ to $55 \Omega$ which would otherwise vary from $43 \Omega$ to $110 \Omega$ over a range of frequency spanning from 0.1 to $9 \mathrm{GHz}$. From Fig. 8(b), it can be observed that for a frequency range spanning from 0.1 to $6.8 \mathrm{GHz}$, the improvement in signal injection efficiency is due to reduced inductance. From approximately 6 to $9 \mathrm{GHz}$, the imaginary part of the input impedance of the original design decreases rapidly from approximately $j 30 \Omega$ and becomes capacitive at around $7.3 \mathrm{GHz}$. As for the proposed design, the imaginary part of the input impedance decreases gradually to close to the purely resistive mark but does not become capacitive. For 


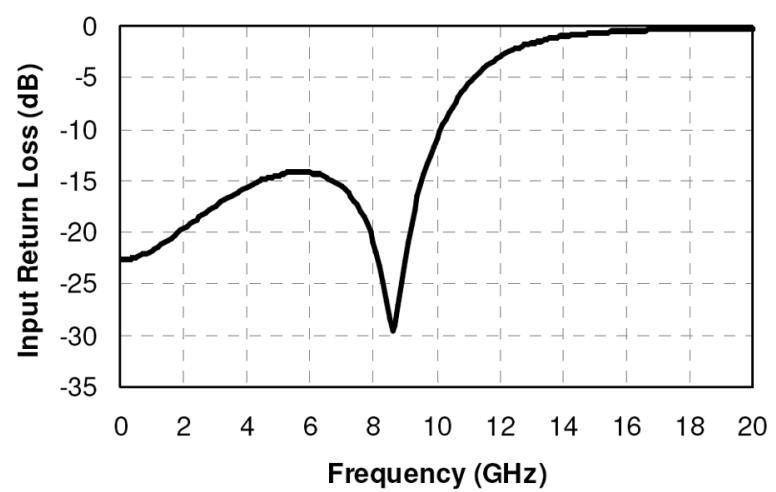

(a)

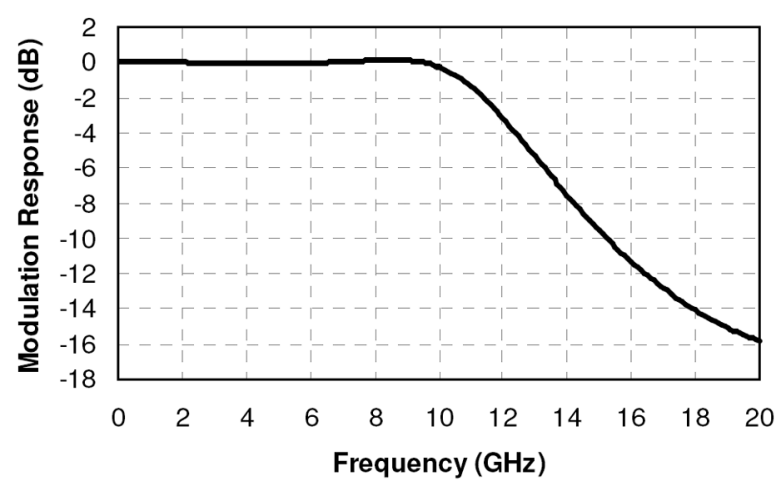

(b)

Fig. 7. (a) Input return loss response and (b) modulation responses of the 10-Gb/s EML module incorporating the microstrip transmission-line implementation of the L-network. The device capacitance of the EAM section was reduced from approximately 0.6 to $0.5 \mathrm{pF}$.

the proposed design, the imaginary part of the input impedance is controlled to within $j 20 \Omega$ to $j 0 \Omega$ instead of within around $j 30 \Omega$ to $-j 50 \Omega$. In a nutshell, the improvement of the signal injection efficiency of the EMLs is due to the well-controlled input impedance brought about by the uniquely implemented L-network. With this method, EMLs can be designed to yield enhanced signal injection efficiency and better optical performance than before.

\section{DEVICE IMPLEMENTATION}

Fig. 9 shows a possible implementation of the proposed EML carrier/submount designs using microstrip transmission-line with ground plane. The coplanar ground plane of which the EML is bonded is connected to the bottom ground plane using vias. On one side of the EML are the conventional 50- $\Omega$ microstrip transmission-line and the uniquely implemented L-network [represented by TL2 in Fig. 5(a)]. On the other side of the EML consists of two 50- $\Omega$ microstrip transmission-lines that act as bond-pads on both ends of the 50- $\Omega$ thin-film termination resistor and a direct-current $(\mathrm{dc})$ bias feed-line.

The value of L1 and L2 should be kept as low as possible to minimize their deteriorating effects on module performance. Hence, the length of the EML carrier/submount was determined to minimize the value of L1 and L2. To achieve reasonably good precision for the value of $\mathrm{L} 3$ and $\mathrm{L} 4$, the repetitive accuracy of the automatic wire-bonder must be carefully evaluated. The choice of bonding-wire would then determine the distance

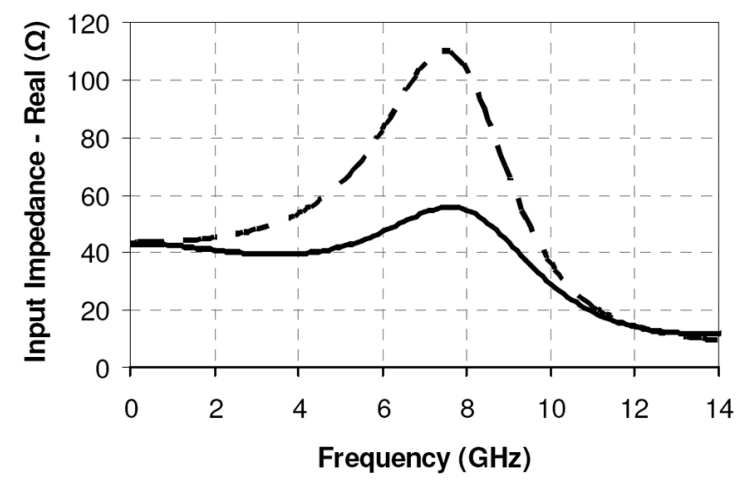

(a)

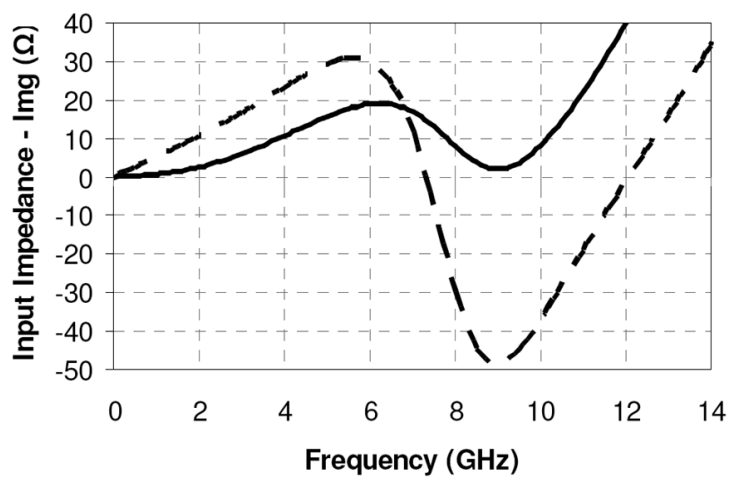

(b)

Fig. 8. Input impedance response of the EML module incorporating a reduced device capacitance of the EAM section (approximately $0.5 \mathrm{pF}$ ) and the microstrip transmission-line implementation of the L-network. (a) Real part and (b) imaginary part. The responses for the proposed design are shown in solidline, whereas the responses in dashed-line belong to that of the typical design shown in Fig. 2(a).

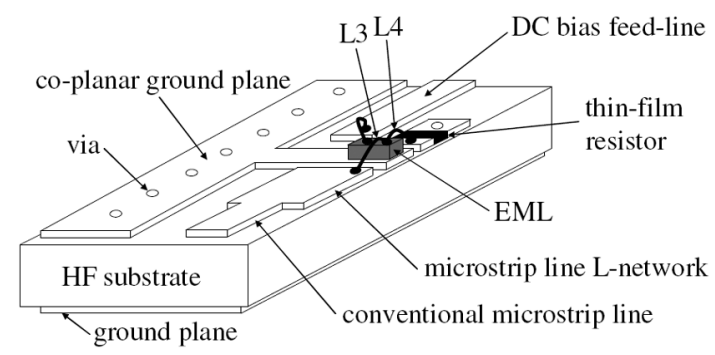

Fig. 9. Possible implementation of the proposed EML carrier/submount designs using microstrip transmission-line with ground plane.

from the microstrip transmission-line L-network and the 50- $\Omega$ thin-film termination resistor to the EML. As a result, the total length of the signal feeder was $3.4 \mathrm{~mm}$. For the responses shown in Fig. 5 and a 0.3 -mm-thick HF substrate having a relative dielectric constant of 8.7, the length and width of the microstrip transmission-line L-network are 1.641 and $0.861 \mathrm{~mm}$, respectively. Generally, the EAM section of EMLs for 10-Gb/s applications is 150 to $200 \mu \mathrm{m}$ long. The continuous-wave DFB LD section does not affect the HF performance of the EML because the isolation resistance between the EAM section and DFB LD section is typically $20 \mathrm{k} \Omega$. Hence, the length of the DFB LD section, design of the dc bias feed-line, and the bonding-wire connecting them have no effect on the HF performance of the EML. Nevertheless, the choice of the length of the DFB LD 


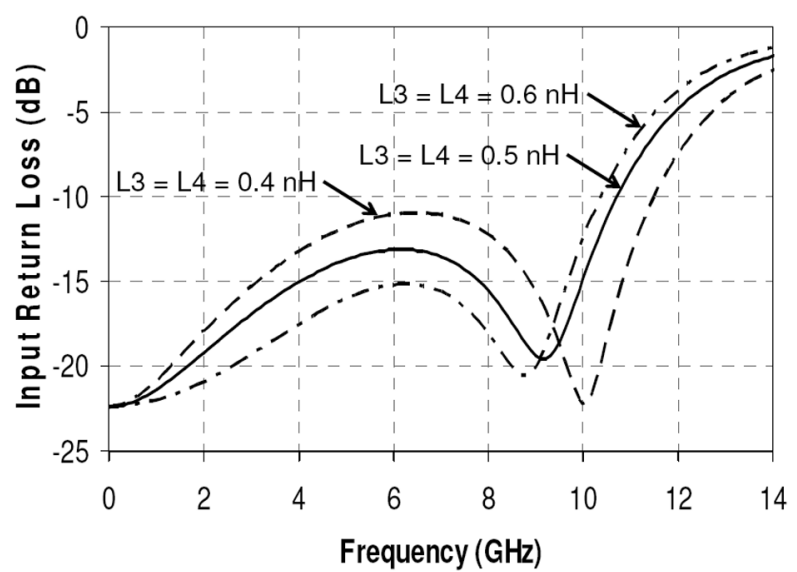

(a)

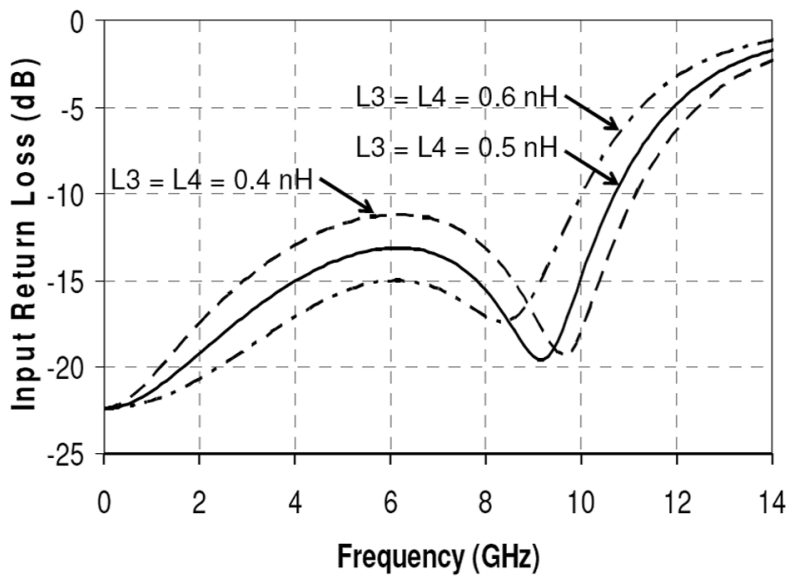

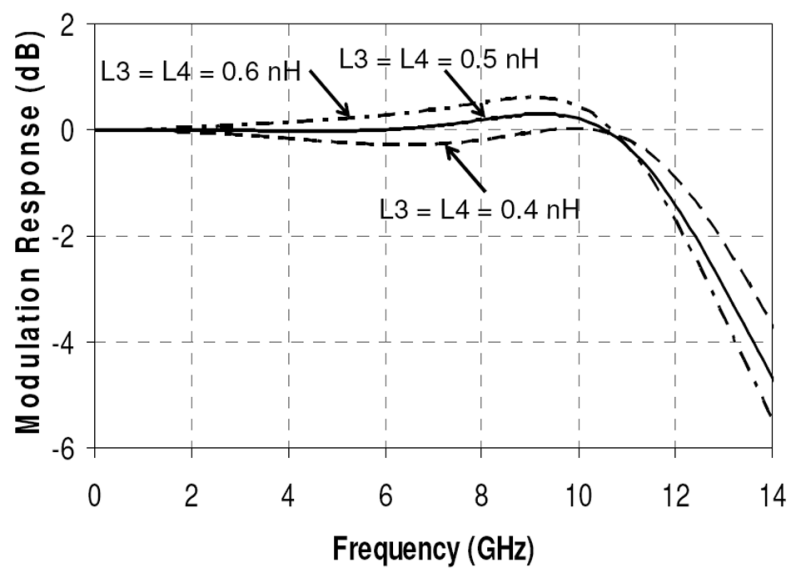

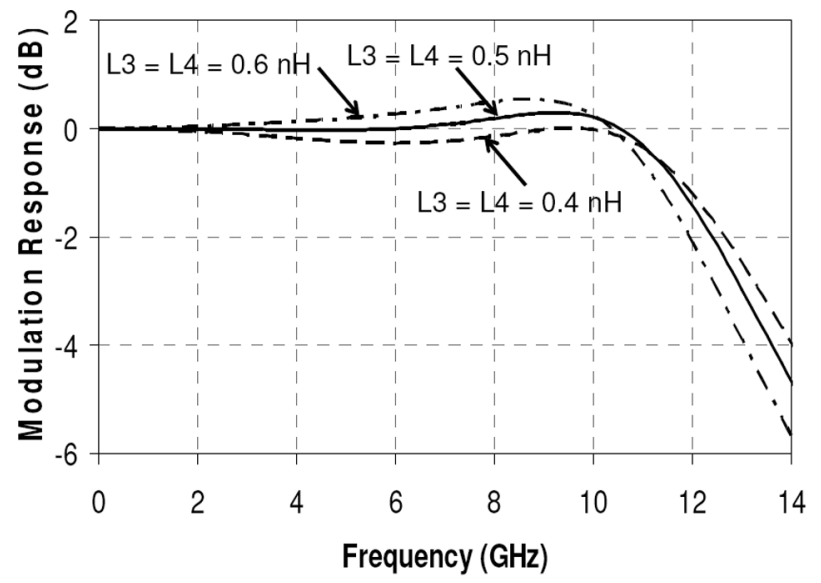

(b)

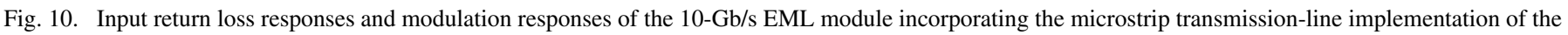

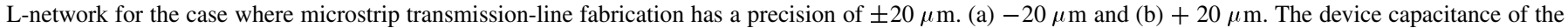
EAM section was $0.45 \mathrm{pF}$.

section determines the maximum optical signal power and the optical signal extinction ratio. Typically, the length of the DFB LD section of $10-\mathrm{Gb} / \mathrm{s}$ EMLs is 300 to $500 \mu \mathrm{m}$.

The robustness of this implementation to fabrication precision was evaluated based on the tolerance of L1, L2, L3, L4, and the microstrip transmission-line (i.e., the conventional 50- $\Omega$ microstrip transmission-line and the proposed microstrip transmission-line L-network). In practice, the value of L1 and L2 can be as low as $0.1 \mathrm{nH}$. However, a value of $0.2 \mathrm{nH}$ have been considered in the evaluation.

\section{TOLERANCE ANALYSIS}

Through a series of simulations, it was found that the proposed implementation can tolerate a fabrication precision of $\pm 20 \mu \mathrm{m}$ for the microstrip transmission-line and $\pm 0.1 \mathrm{nH}$ for L3 and L4. Fig. 10(a) shows the input return loss and modulation responses when the microstrip transmission-line is $-20 \mu \mathrm{m}$ short of design value. When L3 and L4 have an inductance that is $0.1 \mathrm{nH}$ lower than the nominal value of $0.5 \mathrm{nH}$, it was observed from the input return loss responses that the $-10 \mathrm{~dB}$ cross-over frequency was extended from 10.7 to $11.5 \mathrm{GHz}$ and the peak of the hump at around $6 \mathrm{GHz}$ was raised from $-13 \mathrm{~dB}$ to $-11 \mathrm{~dB}$.
On the other hand, the $-10 \mathrm{~dB}$ cross-over frequency was reduced to $10.4 \mathrm{GHz}$ and the peak of the hump at around $6 \mathrm{GHz}$ was lowered to $-15 \mathrm{~dB}$. In either case, the $-3 \mathrm{~dB}$ bandwidth is in excess of $10 \mathrm{GHz}$ (i.e., more than $13 \mathrm{GHz}$ for the former and more than $12 \mathrm{GHz}$ for the latter). The input return loss and modulation responses for the case where the microstrip transmission-line is $20 \mu \mathrm{m}$ in excess of design value are shown in Fig. 10(b). As can be noted from the input return loss responses, the $-10 \mathrm{~dB}$ cross-over frequency extends to $11 \mathrm{GHz}$ and the peak of the hump at around $6 \mathrm{GHz}$ rises to $-11 \mathrm{~dB}$ when $\mathrm{L} 3$ and L4 have an inductance of $0.4 \mathrm{nH}$. For the case where L3 and L4 have an inductance of $0.6 \mathrm{nH}$, the input return loss response showed that the input return loss rises above $-10 \mathrm{~dB}$ at $10 \mathrm{GHz}$ and has a peak of $-15 \mathrm{~dB}$ at around $6 \mathrm{GHz}$. The $-3 \mathrm{~dB}$ bandwidth for the former is more than $13 \mathrm{GHz}$ and more than $12 \mathrm{GHz}$ for the latter. It should be noted that the device capacitance of the EAM section of the EML used in the above harsh evaluation was $0.45 \mathrm{pF}$. The above evaluation is a harsh one because microstrip transmission-line fabrication has a higher precision than that considered here. In actual fact, microstrip transmission-lines can be fabricated very precisely through calibration. Also, by using the appropriate bonding-wires and knowing the capability of the automatic wire-bonder the nominal values of 
L1, L2, L3, and L4 can be achieved. It should be mentioned that the value of $\mathrm{L} 1$ and $\mathrm{L} 2$ that produced the results shown in Fig. 7 was $0.3 \mathrm{nH}$. Hence, some tolerances have already been incorporated in that design because the value of $\mathrm{L} 1$ and $\mathrm{L} 2$ can be as low as $0.1 \mathrm{nH}$ in practice. Therefore, it should be possible to meet the specifications with an EML having an EAM section with a device capacitance of $0.5 \mathrm{pF}$.

\section{CONCLUSION}

To substantially subside the unfavorable effects (i.e., reduced extinction ratio, compromised chirp performance, and lower optical output power) of reducing the device capacitance of the EAM section of the EML to improve the signal injection efficiency of the EML, this work has proposed to incorporate a lumped-element impedance-matching network (L-network) on the LD carrier. To avoid additional cost and reliability issue due to increased component-count, a unique implementation of the L-network was proposed and illustrated. With the L-network incorporated and the device capacitance of the EAM reduced to $0.5 \mathrm{pF}$ (a reduction of about $14 \%$ as oppose to about $33 \%$ in the case of other methods), the signal injection efficiency was found to increase from approximately $60 \%-68 \%$ to $80 \%-97 \%$ (an improvement of approximately 12\%-37\%) over a frequency range spanning from 5.8 to $10 \mathrm{GHz}$. This was achieved at the expense of a reduction in modulation bandwidth from approximately 13 to $12 \mathrm{GHz}$ (a mere $7.69 \%$ for the case where the device capacitance of the EAM is reduced to $0.5 \mathrm{pF}$ ). There are several approaches to apply this impedance-matching technique to higher bit-rates. One of them is to incorporate the driver into the package whereby the EML carrier/submount becomes shorter. If, however, the same package is to be used, HF substrates with a higher dielectric constant should be used. Otherwise, appropriate packages that reduce the length of the EML carrier/submount should be used. Basically, the size of the EML carrier/submount must be reduced in order for this impedance-matching network to support higher data-rates.

\section{ACKNOWLEDGMENT}

The author would like to thank the Chips Implementation Center (CIC) for the loan of the Advanced Design System-a popular and well-established commercial HF computer-aideddesign package.

\section{REFERENCES}

[1] V. Kaman, X. Zheng, S. Yuan, J. Klingshirn, C. Pusarla, R. J. Helkey, O. Jerphagnon, and J. E. Bowers, "A $32 \times 10 \mathrm{~Gb} / \mathrm{s}$ DWDM metropolitan network demonstration using wavelength-selective photonic cross-connects and narrow-band EDFAs," IEEE Photon. Technol. Lett., vol. 17, no. 9, pp. 1977-1979, Sep. 2005.

[2] T. Okiyama, H. Nishimoto, I. Yokota, and T. Touge, "Evaluation of 4-Gbit/s optical fiber transmission distance with direct and external modulation," J. Lightw. Technol., vol. 6, no. 11, pp. 1686-1692, Nov. 1988.

[3] H. Sano, T. Ido, S. Tanaka, and H. Inoue, "10 Gb/s, $80 \mathrm{~km}$ normal fiber transmission by using multiple-quantum well Mach-Zehnder modulator," in Proc. Optoelectron. Commun. Conf., Chiba, Japan, 1996, paper 17D2-4.
[4] O. Mitomi, I. Kotaka, K. Wakita, S. Nojima, K. Kawano, Y. Kawamura, and H. Asai, "40-GHz bandwidth InGaAs/InAlAs multiple quantum well optical intensity modulator," Appl. Opt., vol. 31, pp. 2030-2035, 1992.

[5] M. Aoki, M. Suzuki, H. Sano, T. Kawano, T. Ido, T. Taniwatari, K. Uomi, and A. Takai, "InGaAs/InGaAsP MQW electro-absorption modulator integrated with a DFB laser fabricated by band-gap energy control selective area MOCVD," IEEE J. Quantum Electron., vol. 29, no. 6, pp. 2088-2096, Jun. 1993

[6] T. Kataoka, Y. Miyamoto, K. Hagimoto, K. Wakita, and I. Kotaka, "Ultrahigh-speed driverless MQW intensity modulator, and $20 \mathrm{Gbit} / \mathrm{s} 100$ km transmission experiments," Electron. Lett., vol. 28, pp. 897-898, 1992.

[7] Y. D. Bae, B. K. Kang, B. Park, S. M. Lee, Y. H. Kim, H. K. Kim, M. K. Park, I. Kim, and D. H. Jang, "Operation of 1550-nm electroabsorptionModulated laser at $40^{\circ} \mathrm{C}$ for $10-\mathrm{Gb} / \mathrm{s}, 40-\mathrm{km}$ transmission," IEEE J. Sel. Topics Quantum Electron., vol. 11, no. 1, pp. 135-140, Jan.-Feb. 2005.

[8] I. Kim, B. K. Kang, Y. D. Bae, B. Park, S. M. Lee, Y. H. Kim, and D. H. Jang, "Design of amplifier- and modulator-integrated laser diode for 10-Gb/s 80-km transmission," IEEE J. Sel. Topics Quantum Electron., vol. 11, no. 2, pp. 323-328, Mar.-Apr. 2005.

[9] T. Ido, H. Sano, D. J. Moss, S. Tanaka, and A. Takai, "Strained InGaAs/ InAlAs MQW electroabsorption modulators with large bandwidth and low driving voltage," IEEE Photon. Technol. Lett., vol. 6, no. 10, pp. 1207-1209, Oct. 1994.

[10] E. Ishimura, Y. Miyazaki, and M. Kawano, "Light modulator module and method for fabricating light modulator module," U.S. patent 5,602,672, Feb. 1997.

[11] C. G. Lim, "The effect of signal-feeder characteristic impedance on the signal injection efficiency of electro-absorption modulator integrated lasers," J. Lightw. Technol., vol. 24, no. 10, pp. 3835-3841, Oct. 2006.

[12] S. H. Lee, J. H. Ahn, Y. K. Oh, J. S. Ma, A. G. Choo, T. I. Kim, Y Kim, and J. Jeong, "High performance modules of 2.5 Gbps modulator integrated DFB lasers using new RF impedance matching technique," IEEE Trans. Adv. Packag., vol. 24, no. 3, pp. 407-410, Aug. 2001.

[13] D. M. Pozar, Microw. Eng. Hoboken, NJ: Wiley, 2000.

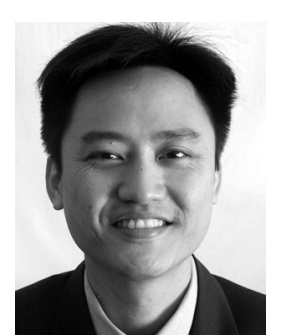

Cheng Guan Lim (S'94-A'97-M'00) received the B.Eng. (honors) degree in electronic and electrical engineering in 1997 and the Ph.D. degree at the Institute of Microwaves and Photonics, School of Electronic and Electrical Engineering, University of Leeds, Leeds, U.K., in 2001.

From 2001 to 2003, he was a Research Engineer at the Yokohama Research and Development Laboratories, Furukawa Electric Company, Yokohama, Japan, working on the development of semiconductor laser modules for digital and analog telecommunication applications. In 2003, he joined Agilent Technologies, Singapore, as a Senior Engineer focusing on optoelectronic device manufacturing. From 2004 to 2005, he was a Senior Research Scientist at the Institute for Infocomm Research, Singapore (a member of the Agency for Science, Technology, and Research, Singapore, and a National University of Singapore affiliated research institute) dealing with radio-over-fiber technologies. He was an Assistant Professor at the Department of Photonics/Institute of Electro-Optical Engineering, National Chiao Tung University, Hsinchu, Taiwan, R.O.C. Currently, he is a Researcher at the National Institute of Advanced Industrial Science and Technology, Tsukuba, Japan, where he conducts research on intersubband transitions in InGaAs/AlAs/AlGaAsSb-coupled double-quantum-wells. His prime research interests include the investigation of the fundamental physics of advanced nanostructures and the optical and electronic properties of advanced novel materials through simulation and experimentation. His secondary research interests are photonic packaging, RF photonics, and photonic integrated circuits. He has one granted U.S. patent, written one invention disclosure, and published several international refereed journal and conference papers.

Dr. Lim has served as a Steering Committee Member for the Inaugural IEEE International Workshop on Antenna Technology 2005 (Singapore), and as a Technical Program Committee member for the 2005 Symposium on Technology Fusion of Optoelectronics and Communications-International Conference on Photonics (Taiwan, R.O.C.). In 1995, he was awarded the Crab Tree Prize for outstanding academic achievement, the Hewlett-Packard Prize in 1997 for the recognition as the best student in High-Frequency Engineering, and the F. W. Carter Prize in 2001 for presenting the best Ph.D. dissertation. His biographical profile was selected for inclusion in the 25th Silver Anniversary Edition of Who's Who in the World. 